\title{
Leveraging psychiatric and medical genetics to understand comorbid depression and obesity ${ }^{\dagger}$
}

Anna R. Docherty

\section{Summary}

Precision medicine in psychiatry is on the rise, and depression and obesity - two highly prevalent, comorbid and well-characterised phenotypes - are optimal targets for the approach. Add the bedrock susceptibility gene, FTO, and Riviera et al have identified a constellation of factors that could enhance clinical treatment of both disorders.

\section{Declaration of interest \\ None.}

\section{Copyright and usage}

(c) The Royal College of Psychiatrists 2017.
Anna R. Docherty is Assistant Professor of Psychiatry at the University of Utah and the Virginia Commonwealth University. Her research integrates dimensional phenotypic assessment and genomic data to predict risk for severe psychopathology. She explores strategies for genetic subtyping and risk analysis, and the influences of comorbid conditions on psychiatric trajectories

\section{Integrating mental and physical health factors to inform public health}

Psychiatric disorders are difficult to characterise using biological methods because they are genetically very complex and heterogeneous. Although 'precision medicine' in a non-psychiatric context involves, for example, monogenic subtypes of breast cancer or leukaemia that lead to a personalised drug target, precision medicine in psychiatry is considerably more challenging. The hundreds of genetic variants conferring a small proportion of psychiatric risk precludes simpler candidate gene approaches to enhancing treatment. The Psychiatric Genomics Consortium has begun addressing this problem of psychiatric polygenicity in precision medicine in a number of ways, including an integration of omics approaches with other biological and bioinformatics methods. ${ }^{1}$ With these efforts in mind, it is additionally important to consider the utility of information derived from the medical genetics of conditions frequently co-occurring with psychiatric phenotypes.

Psychiatric disorders, although often comorbid with other conditions, are not typically studied by genetics researchers against a backdrop of health factors. One elegant approach to reducing the genetic heterogeneity of psychiatric disorders (e.g. major depressive disorder) is to account for medical (somatic) genetic variation. For example, unlike depression or schizophrenia, obesity studies have produced reliable genetic variants that account for significant elevation in body mass index (BMI) and these variants may interact with psychiatric status to increase risk. The accompanying article provides one such example of a medical genetics integration: here, Rivera and colleagues examine the interaction of depression and FTO, a well-validated obesity susceptibility gene, on BMI. ${ }^{2}$ They consider how the wellestablished comorbid relationship of high BMI and major depressive disorder ${ }^{3,4}$ may be moderated by FTO.

†'see pp. 70-76, this issue.
To this end, the authors analyse 6902 cases with depression and 6799 controls from three replication cohorts and two original discovery cohorts, observing a significant interaction between FTO, BMI and major depression in a fixed effects meta-analysis ( $\beta=0.12, \quad P=2.710-4$ ) of replication samples (with further support from analyses of the full, combined sample). Their results indicate that carriers of the risk allele who have depression have a $2.2 \%$ increase in BMI for each risk allele over and above the main effect of FTO. These findings have tangible implications for BMI-related health risks (e.g. diabetes, cardiovascular disease, early cognitive decline) in genetic subsets of patients with major depression symptoms - patients who are relatively easy to identify and carry elevated risk for other psychiatric disorders. In this, they demonstrate how data-driven biomedical research, integrated with psychiatric genetics, can move us towards informing and improving clinical practice.

\section{Steps towards a more targeted approach to genetic risk assessment}

Depression and obesity - two of the most prevalent, impactful, well-characterised and expensive public health problems - are prime candidates for precision medicine. Depressive symptoms and increased BMI frequently co-occur, and their causation is observed to be bi-directional and reciprocal. ${ }^{3}$ Both phenotypes have considerable potential to stratify risk in the other and both are independently associated with significant long-term health risks. Add to this a bedrock susceptibility gene that has demonstrated associations with obesity and depression, and Riviera et al have identified a constellation of predictive factors for both disorders.

The genetic study is characterised by various strengths, including access to large sample sizes and the use of appropriate analytical methods. Critically, an ideal candidate gene was chosen for BMI analyses: this polymorphism has been associated with significant elevations in BMI across numerous studies and across multiple ancestry groups. And the findings may have implications for psychiatric outcomes: over $80 \%$ of those with serious mental illness (SMI) are overweight or obese, SMI patients exhibit mortality rates of two to three times that of the general population, and that mortality is largely accounted for by cardiovascular disease. ${ }^{5}$ Although antipsychotic medication is one culprit, significant factors over and above medication are 
poor diet and sedentary lifestyle, and these have been targeted successfully in SMI with weight loss counselling and education. ${ }^{6,7}$

Limitations to this research include the presence of variation in the ascertainment of depression cases across individual studies, and the lack of information relating to other psychiatric or medical comorbidities. Differences in the ascertainment strategy across the studies may have contributed to variation in the significance of the findings observed across these three replication samples. Similarly, Rivera and colleagues reach mixed conclusions across the fixed and random effects estimators in their replication meta-analysis, which may suggest some limitation to the robustness of the findings. Finally, examination of comorbid psychiatric disorders could have improved signal.

\section{Expanding the current methods to enhance psychiatric genomics}

As this type of data is increasingly available via electronic medical records and national health registries, causality in this type of study should be more carefully considered. Broadly, a causal relationship between these factors cannot be assumed. The facts here, as is so often the case in observational research, are merely that all three factors - BMI, depression and FTO genotype - are correlated. However, here, unlike in other observational research, we have compelling biochemical prior information linking FTO to obesity, and this information could be used to (tentatively) identify the direction of moderation (i.e. that depression ${ }^{\star} F T O$ leads to obesity). Moreover, one could empirically scrutinise causal models by using structural equation modelling and instrumental variables or running this analysis on a backdrop of polygenic risk for obesity and depression based on genome-wide association study summary statistics. Thus, it remains for future research to exploit the opportunity to use statistical modelling and prior information on the biochemical mechanisms of FTO to adjudicate between potential causal models.

Precision medicine in psychiatry will continue to flesh out causality by testing a variety of environment moderation models. These models will eventually integrate genome-wide polygenic risk metrics, use more sophisticated, dimensional approaches to measuring psychopathology, and reap the massive advantages of electronic medical record data by way of machine learning methods. ${ }^{8-10}$ The benefit of integrating polygenic risk metrics into these models will likely be considerable. Other much needed research relates to modelling patterns of depression, FTO and
BMI across other major predictors of obesity and cardiovascular risk, such as gender, ancestry, age at menarche and socioeconomic status.

It is widely understood that psychopathology has a broad and deep impact on physical health outcomes, but psychiatry, like genetics, has yet to fully actualise its promise in the clinic. The current report by Rivera et al is a step towards both precision medicine and precision psychiatry, and a simple and elegant rendition of important things to come.

Anna R. Docherty, PhD, Department of Psychiatry, University of Utah School of Medicine, 383 Colorow Way \#338, SLC, Utah 84110, USA. Email: anna.docherty@ utah.edu

First received 2 Dec 2016, final revision 30 Mar 2017, accepted 8 May 2017

\section{References}

1 Sullivan PF, Agrawal A, Bulik CM, Andreassen OA, Borglum AD, Breen G, et al. Psychiatric genomics: an update and an agenda. bioRxiv 2017 (preprint $10 \mathrm{Mar}$, https://dx.doi.org/10.1101/115600).

2 Rivera $M$, Locke $A E$, Corre $T$, Czamara $D$, Wolf $C$, Ching-Lopez A, et al. Interaction between the FTO gene, body mass index and depression: meta-analysis of 13701 individuals. Br J Psychiatry 2017; 211: 70-6.

3 Luppino FS, de Wit LM, Bouvy PF, Stijnen T, Cuijpers P, Penninx BW, et al. Overweight, obesity, and depression: a systematic review and meta-analysis of longitudinal studies. Arch Gen Psychiatry 2010; 67: 220-9.

4 Peng S, Zhu Y, XU F, Ren X, Li X, Lai M. FTO gene polymorphisms and obesity risk: a meta-analysis. BMC Med 2011; 9: 71.

5 McGinty EE, Baller J, Azrin ST, Juliano-Bult D, Daumit GL. Interventions to address medical conditions and health-risk behaviors among persons with serious mental illness: a comprehensive review. Schizophr Bull 2016; 42: 96-124.

6 Daumit GL, Dickerson FB, Wang NY, Dalcin A, Jerome GJ, Anderson CA, et al. A behavioral weight-loss intervention in persons with serious mental illness. N Engl J Med 2013; 368: 1594-602.

7 Teasdale SB, Ward PB, Rosenbaum S, Samaras K, Stubbs B. Solving a weighty problem: systematic review and meta-analysis of nutrition interventions in severe mental illness. Br J Psychiatry 2017; 210: 110-8.

8 Krueger RF, Tackett JL, MacDonald A. Toward validation of a structural approach to conceptualizing psychopathology: a special section of the Journal of Abnormal Psychology. J Abnorm Psychol 2016; 125: 1023-6.

9 Adkins DE. Machine learning and electronic health records: a paradigm shift. Am J Psychiatry 2017; 174: 93-4.

10 Hoffmann TJ, Ehret GB, Nandakumar P, Ranatunga D, Schaefer C, Kwok PY, et al. Genome-wide association analyses using electronic health records identify new loci influencing blood pressure variation. Nat Genet 2017; 49 $54-64$. 